\title{
Development and validation of an LC-MS/MS method after chiral derivatization for the simultaneous stereoselective determination of methylenedioxy-methamphetamine
} (MDMA) and its phase I and II metabolites in human blood plasma

\author{
Andrea E. Steuer ${ }^{1}$, Corina Schmidhauser ${ }^{1}$, Matthias E. Liechti ${ }^{2}$ and Thomas Kraemer ${ }^{1}$ \\ ${ }^{1}$ Department of Forensic Pharmacology \& Toxicology, Zurich Institute of Forensic Medicine, \\ University of Zurich, Switzerland \\ ${ }^{2}$ Psychopharmacology Research, Division of Clinical Pharmacology and Toxicology, \\ Department of Biomedicine and Department of Clinical Research, University Hospital Basel, \\ Basel, Switzerland
}

This is the peer reviewed version of the following article: Steuer, A. E., Schmidhauser, C., Liechti, M. E., and Kraemer, T. (2015), Development and validation of an LC-MS/MS method after chiral derivatization for the simultaneous stereoselective determination of methylenedioxy-methamphetamine (MDMA) and its phase I and II metabolites in human blood plasma. Drug Test. Analysis, 7, 592-602, which has been published in final form at http://dx.doi.org/10.1002/dta.1740. This article may be used for non-commercial purposes in accordance with Wiley Terms and Conditions for Self-Archiving.

*Corresponding author:

Dr. Andrea E. Steuer

University of Zurich

Zurich Institute of Forensic Medicine

Department of Forensic Pharmacology \& Toxicology

Winterthurerstrasse 190/52

CH-8057 Zurich

Switzerland

Tel.: 0041446355679 ; fax: 0041446356852

E-mail address: andrea.steuer@irm.uzh.ch (A. Steuer) 


\section{ABSTRACT}

3,4-Methylenedioxymethamphetamine (MDMA, ecstasy) is a racemic drug of abuse and its two enantiomers are known to differ in their dose-response curves. The $S$-enantiomer was shown to be eliminated at a higher rate than the $R$-enantiomer. The most likely explanation for this is a stereoselective metabolism also claimed in in vitro studies. Urinary excretion studies showed that the main metabolites in humans are 4-hydroxy 3-methoxymethamphetamine (HMMA) 4-sulfate, HMMA 4-glucuronide and 3,4-dihydroxymethamphetamine (DHMA) 3sulfate. For stereoselective pharmacokinetic analysis of these phase II metabolites in human blood plasma useful analytical methods are needed. Therefore the aim of the presented study was the development and validation of a stereoselective LC-MS/MS method for the simultaneous quantification of MDMA, 3,4-methylenedioxyamphetamine, DHMA, DHMA 3sulfate, HMMA, HMMA 4-glucuronide, HMMA 4-sulfate, and 4-hydroxy 3methoxyamphetamine in blood plasma for evaluation of the stereoselective pharmacokinetics in humans. Blood plasma samples were prepared by simple protein precipitation and afterwards all analytes were derivatized using $N$-(2,4-dinitro-5-fluorophenyl) L-valinamide resulting in the formation of diastereomers which were easily separable on standard reverse phase stationary phases. This simple and fast method was validated according to international guidelines including specificity, recovery, matrix effects, accuracy and precision, stabilities, and limits of quantification. The method proved to be selective, sensitive, accurate and precise for all tested analytes except for DHMA. The method was applied to the analysis of more than 400 samples from a controlled study after application of MDMA.

Keywords: chiral derivatization, LC-MS/MS analysis, MDMA, phase II metabolites, human blood plasma 


\section{Introduction}

3,4-Methylenedioxymethamphetamine (MDMA, ecstasy) is an illicit drug of abuse that possesses in addition to its amphetamine-like stimulant effects also hallucinogenic properties, leading to feelings of energy, friendliness, euphoria and empathy [1-4]. After decreasing numbers of MDMA seizures in recent years, most likely due to its non-availability on the illicit drug market, the Substance Abuse and Mental Health Services Administration and the European Monitoring Centre for Drugs and Drug Addiction (EMCDDA) have reported on increasing MDMA consumption in the United States and Europe again since 2010 [5, 6]. Consumption of MDMA may lead to severe acute poisonings including symptoms such as tachycardia, hypertension, hyperthermia, or serotonin syndrome [1, 2]. Neurotoxic effects to serotonic neurons have been described, but are still controversial discussed in terms of species and dosing [7-9]. MDMA metabolism is suspected to be responsible for neurotoxicity presumably through the formation of glutathion adducts [10-16].

Figure 1 describes the main metabolic pathways of MDMA observed in humans. The major pathway includes $O$-demethylenation to 3,4-dihydroxymethamphetamine (DHMA), followed by $O$-methylation mainly to 4-hydroxy-3-methoxymethamphetamine (HMMA). DHMA is mainly further sulfated by sulfotransferases to DHMA 3-sulfate and DHMA 4-sulfate. HMMA can be further conjugated by UDP-glucuronyltransferases or by sulfotransferases. A minor pathway includes demethylation to 3,4-methylendioxyamphetamine (MDA) followed by demethylenation to 3,4-dihydroxyamphetamine, $O$-methylation to 4-hydroxy-3methoxyamphetamine (HMA) and conjugation [17-19]. In urine samples of recreational MDMA users following a controlled single dose of MDMA, DHMA 3-sulfate, HMMA 4sulfate and HMMA 4-glucuronide were detected as major metabolites next to unchanged MDMA [20].

Chemically, MDMA is a ring-substituted phenylalkylamine derivative that possesses a chiral center. Different properties regarding pharmacological effects, neurotoxicity and in vivo 
kinetics for the two enantiomers $R$ - and $S$-MDMA are described [1, 2, 21-24]. The $S$ enantiomer is eliminated at a higher rate than the $R$-enantiomer $[1,2,21-24]$ most likely explained by stereoselective metabolism as claimed in various vitro experiments [25-28]. In order to perform systematic pharmacokinetic studies on the stereoselectivity in vivo, respective analytical methods are needed. So far, the most commonly used instrumental technique for chiral analysis of MDMA and metabolites, is gas chromatography-mass spectrometry (GC-MS). Mainly derivatization with different chiral derivatization reagents is employed, leading to the formation of diastereomers which can be separated by achiral chromatography methods [25, 29-32]. All these methods require hydrolysis procedures of glucuronides and sulfates. However, for complete investigation of stereoselectivity in MDMA metabolism monitoring the intact phase II metabolites is of major importance. Methods, especially stereoselective ones, for phase II metabolites of MDMA are scarce in the literature. Shima et al published an LC-MS method for detection and quantification of HMMA sulfate and glucuronide in human urine [33], but only for the racemates. Schwaninger et al. aimed to stereoselectively determine all relevant phase II metabolites in human urine, however, three different methods employing GC-MS and LC-MS were necessary to analyze all metabolites in a stereoselective manner [34]. Recently, Nakanishi et al described a new LC-MS method after chiral derivatization with $N$-(2,4-dinitrophenyl)-D-leucinamide for stereoselective analysis of MDMA as well as of HMMA sulfate and HMMA glucuronide in urine, but unfortunately this method did not cover DHMA sulfates [35]. To the best of our knowledge no stereoselective methods for detection and quantification of MDMA and all relevant phase II metabolites in blood plasma are available at the moment. Therefore, the aim of the presented study was the development and validation of a stereoselective method for simultaneous analysis of MDMA and all relevant phase I and phase II metabolites in human blood plasma. This method should be used for the analysis of blood plasma samples from a double-blind, placebo-controlled, crossover study with $125 \mathrm{mg}$ of MDMA. 


\section{Experimental}

\section{Chemicals and reagents}

Hydrochlorides of racemic MDA, HMA, MDMA, HMMA and DHMA and methanolic solutions (1 mg/mL) of MDA-d5 and MDMA-d5 were obtained from Lipomed (Arlesheim, Switzerland). 4-hydroxymethamphetamine (pholedrine), 3,4-dihydroxybenzylamine (DHBA) and the derivatization reagent $N$-(2,4-dinitro-5-fluorophenyl) L-valinamide (DNPV) were from Sigma Aldrich (Buchs, Switzerland). R/S-DHMA sulfates, $R / S$-HMMA 4-sulfate and single diastereomers of HMMA 4-glucuronides were synthesized as described in refs.[28, 36]. $S$-MDMA was a kind gift of The Department of Experimental and Clinical Pharmacology and Toxicology, Saarland University, Germany and had been prepared through enantioseparation of racemic MDMA as described in ref. [25]. Water was purified with a Millipore filtration unit and acetonitrile (ACN) and methanol of HPLC grade were obtained from Fluka (Buchs, Switzerland). All other chemicals used were from Merck (Zug, Switzerland) and of the highest grade available.

\section{LC-MS/MS Method Development}

\section{Sample preparation for method development}

To $50 \mu \mathrm{L}$ of each analyte solution $(100 \mu \mathrm{M}$ each) $100 \mu \mathrm{L}$ of carbonate buffer $(1 \mathrm{M}, \mathrm{pH} 9)$ and $100 \mu \mathrm{L}$ of DNPV ( $0.3 \%$ in acetone) were added and the mixture was left in a heating block for $30 \mathrm{~min}$ at $50^{\circ} \mathrm{C}$. Afterwards the reaction was stopped by the addition of $20 \mu \mathrm{L} 1 \mathrm{M} \mathrm{HCl}$. Finally, $180 \mu \mathrm{L}$ of a mixture of mobile phase A and B (1:1, v/v, see below) were added and aliquots of $10 \mu \mathrm{L}$ of this solution were injected into the LC-MS/MS system.

\section{Optimization of MS parameters}

The analysis was performed using a Thermo Fischer Ultimate 3000 UHPLC (Thermo Fisher, San Jose, California, USA) system coupled to an ABSciex 5500 QTtrap linear ion trap (LIT) 
quadrupole mass spectrometer (ABSciex, Darmstadt/Germany). The Turbo V ion source was operated in positive ESI mode with the following MS conditions: gas 1, nitrogen (50 psi); gas 2, nitrogen (60 psi); ion spray voltage, 4500 ; ion-source temperature, $550{ }^{\circ} \mathrm{C}$; curtain gas, nitrogen (30 psi), collision gas, medium. The MS was operated in the enhanced product ion (EPI) scan mode using the following settings: mass range 50-1000, scan rate 10,000 Da/s, dynamic fill time.

EPI scans were recorded for the expected protonated molecular ion of the potentially underivatized and of the one-, two-, and threefoldly derivatized analytes, respectively. Two runs were performed for each substance, first with collision energy (CE) set to $10 \mathrm{eV}$, second with a $\mathrm{CE}$ of $40 \mathrm{eV}$.

\section{Chromatography optimization}

Different mobile phases were tested on a Phenomenex (Aschaffenburg, Germany) Kinetex C18 column $(100 \times 2.1 \mathrm{~mm}, 2.6 \mu \mathrm{m})$ for the stereoselective separation of all analytes. First experiments were performed using gradient elution with $25 \mathrm{mM}$ ammonium acetate buffer in water containing $0.1 \%(\mathrm{v} / \mathrm{v})$ acetic acid (A) and acetonitrile containing $0.1 \%(\mathrm{v} / \mathrm{v})$ acetic acid (B) at a flow rate of $0.5 \mathrm{~mL} / \mathrm{min}$. Secondly a mobile phase composition of $5 \mathrm{mM}$ ammonium formate in water containing $0.1 \%(\mathrm{v} / \mathrm{v})$ formic acid (C) and $5 \mathrm{mM}$ ammonium formate in methanol containing $0.1 \%(\mathrm{v} / \mathrm{v})$ formic acid (D) at a flow rate of $0.4 \mathrm{~mL} / \mathrm{min}$ were tested. Further experiments were conducted using eluent $\mathrm{C}$ and changing mobile phase $\mathrm{D}$ to ACN containing $0.1 \%(\mathrm{v} / \mathrm{v})$ formic acid $(\mathrm{E})$ or a mixture of methanol/ACN $(3: 1, \mathrm{v} / \mathrm{v})$ containing $0.1 \%$ formic acid $(\mathrm{F})$.

\section{Determination of elution order of $R$ and $S$ enantiomers}

Elution order of $R$ and $S$ enantiomers of MDA, DHMA, HMMA, DHMA 3-sulfate, DHMA 4sulfate and HMMA 4-sulfate were evaluated using incubations of $S$-MDMA with human liver 
S9 fraction (HLS9) as generally described in ref. [26]. Elution order of MDMA and HMMA glucuronide was determined by injection of a derivatized sample of the single $S$-stereoisomer $[25,28]$. For determination of the elution order of HMA, additionally racemic MDA was incubated with HLS9.

\section{Final Procedure for LC-MS/MS analysis}

\section{Blood plasma samples}

Human blood samples (blank) were obtained from 10 different volunteers of the Zurich Institute of Forensic Medicine as lithium heparine blood. Blood plasma was obtained after centrifugation at $5,000 \mathrm{~g}$ for 15 minutes and stored at $-20{ }^{\circ} \mathrm{C}$ until further usage.

\section{Sample Preparation and chiral derivatization of blood plasma}

The plasma samples were prepared by simple protein precipitation. Briefly, to $200 \mu \mathrm{L}$ plasma mixed with $20 \mu \mathrm{L}$ of the internal standard (IS) mixture (MDMA-d5, MDA-d5, pholedrine, DHBA, $2.5 \mu \mathrm{M}$ each) $1000 \mu \mathrm{L}$ of acetonitrile were added; the mixture was shaken and centrifuged $(10,000 \mathrm{~g}, 5 \mathrm{~min})$. An aliquot of $1000 \mu \mathrm{L}$ was transferred into an autosampler vial, $50 \mu \mathrm{L}$ of formic acid were added and the mixture was evaporated to dryness under a gentle stream of nitrogen at $40^{\circ} \mathrm{C}$. The residue was dissolved in $100 \mu \mathrm{L}$ of carbonate buffer $(1 \mathrm{M}$, $\mathrm{pH} 9) .100 \mu \mathrm{L}$ of DNPV (0.3\% in acetone) were added and the mixture was left in a heating block for $30 \mathrm{~min}$ at $50^{\circ} \mathrm{C}$. Afterwards the reaction was stopped by the addition of $20 \mu \mathrm{L} 1 \mathrm{M}$ $\mathrm{HCl}$. Finally, $80 \mu \mathrm{L}$ of a mixture of mobile phase A and B (1:1, v/v) were added and aliquots of $10 \mu \mathrm{L}$ of this solution were injected into the LC-MS/MS system.

\section{Apparatus}


The analysis was performed using a Thermo Fischer Ultimate 3000 UHPLC system (Thermo Fisher, San Jose, California, USA) coupled to an ABSciex 5500 QTtrap linear ion trap (LIT) quadrupole mass spectrometer (ABSciex, Darmstadt/Germany).

The LC settings were as follows: Phenomenex (Aschaffenburg, Germany) Kinetex C18 column $(100 \times 2.1 \mathrm{~mm}, 2.6 \mu \mathrm{m})$, gradient elution with $25 \mathrm{mM}$ ammonium acetate buffer in water containing $0.1 \%(\mathrm{v} / \mathrm{v})$ acetic acid (A) and acetonitrile containing $0.1 \%(\mathrm{v} / \mathrm{v})$ acetic acid (B). The flow rate was $0.5 \mathrm{~mL} / \mathrm{min}$ with the following gradient: $0-2 \mathrm{~min} 2 \% \mathrm{~B}, 2-25 \mathrm{~min}$ to $60 \% \mathrm{~B}, 25-26$ min hold at $60 \% \mathrm{~B}, 26-27 \mathrm{~min}$ to $100 \% \mathrm{~B}, 27-29 \mathrm{~min}$ hold at $100 \% \mathrm{~B}$ and at $29.01 \mathrm{~min}$ reequilibrating to $2 \% \mathrm{~B}$ for $2 \mathrm{~min}$.

The Turbo V ion source was operated in positive ESI mode with the following MS conditions: gas 1 , nitrogen (50 psi); gas 2 , nitrogen (60 psi); ion spray voltage, 4500 ; ion-source temperature, $550{ }^{\circ} \mathrm{C}$; curtain gas, nitrogen $(30 \mathrm{psi})$, collision gas, medium. The MS was operated in the multiple reaction monitoring (MRM) mode using 3 transitions for each analyte except for the ISs where 1 MRM transition was applied. The MS settings for each analyte are given in Table 1.

\section{Data analysis}

Analyst software (1.6.2) was used for peak integration and quantification of data.

\section{Method Validation}

Preparation of calibration and quality control (QC) samples

Separate stock solutions $(1 \mathrm{mM})$ of each racemic analyte and for the single diastereomers of HMMA 4-glucuronide were prepared in water/acetonitrile. Working solutions (1, 10, 50, 100, $200 \mathrm{mM}$ ) were prepared by dilution from each stock solution. Spiking solutions for calibration standards and QC samples were prepared by mixing appropriate amounts of the 
corresponding stock or working solution to obtain concentrations ten times higher than the corresponding blood plasma concentration. All solutions were stored in aliquots at $-20^{\circ} \mathrm{C}$. Calibration standards and QC samples (LOW, MED, HIGH) were prepared from $200 \mu \mathrm{L}$ analyte-free blood plasma and $20 \mu \mathrm{L}$ of the corresponding fortifying solution. The final calibration and QC concentrations are given in Table 2 for each stereoisomer.

\section{Selectivity and cross talk}

$50 \mu \mathrm{L}$ of each analyte solution and each IS $(100 \mu \mathrm{M})$ were derivatized separately as described above and analyzed for interferences in the other MRM transitions.

\section{Specificity}

Ten blank blood plasma samples from different sources were analyzed for peaks interfering with the detection of analytes or IS. Two zero samples (blank sample + IS) were analyzed to check for appropriate IS purity and presence of native analytes.

\section{Recovery and matrix effects}

Recovery (RE) and matrix effect (ME) were determined at QC LOW and HIGH concentration using 6 different blood plasma sources according to the simplified approach described by Matuszewski et al [37].

\section{Calibration model}

Replicates $(n=6)$ at each concentration level were analyzed as described above. The regression lines were calculated using non-weighted, a weighted $[1 / \mathrm{X}]$, and a weighted $[1 / \mathrm{X} 2]$ leastsquares regression models. The final choice of model was made after calculating validation data using these alternatives. Daily calibration curves (single measurement per level) were prepared with each batch of validation samples. The back-calculated concentrations of all 
calibration samples were compared to their respective nominal values and quantitative accuracy was required within $30 \%$ of target.

\section{Accuracy and precision}

QC samples (LOW, MED, HIGH) were analyzed according to the procedures described above in duplicate on each of eight days. Accuracy was calculated in terms of bias as the percent deviation of the mean calculated concentration at each concentration level from the corresponding theoretical concentration. Intra-day and inter-day precision were calculated as relative standard deviation (RSD) according to ref. [38].

\section{Stability}

Processed sample and freeze-thaw stability were investigated at QC LOW and HIGH concentration ( $\mathrm{n}=6$ each) according to ref. [38]. Long-term stability experiments for phase II metabolites were performed after 24 months at QC LOW and HIGH concentration $(n=6$, each), calculated via a freshly prepared calibration curve and accuracy was compared to nominal concentrations.

\section{Limits}

The lowest point of the calibration curve was defined as the limit of quantification (LOQ) of the method and fulfilled the requirement of LOQ, signal to noise ratio of 10:1 determined via peak heights. The limit of detection (LOD) was not systematically investigated.

\section{Proof of applicability}

Blood plasma samples from a double-blind, placebo-controlled, crossover study with $125 \mathrm{mg}$ of MDMA administered to healthy volunteers were assayed with the described method. 


\section{Results and discussion}

\section{LC-MS/MS Method Development}

The $S$-enantiomer of MDMA is eliminated at a higher rate than the $R$-enantiomer $[1,2,21-24]$ most likely explained by stereoselective metabolism that was observed in various in vitro experiments [25-28]. So far, chiral pharmacokinetic studies in blood mainly focused on MDMA and MDA $[22,39]$ and some also on DHMA and HMMA, but only after conjugate cleavage $[24,30]$. Stereoselective differences could be seen for MDMA with preference for the $R$-enantiomer. For the metabolites, slight preferences for $S$-stereoisomers were observed but not to the same extent as for MDMA. Systematic chiral pharmacokinetic studies of all relevant phase I and II metabolites are still missing in the literature. Elimination studies in human urine following controlled administration of MDMA showed, that in fact HMMA 4sulfate, HMMA 4-glucuronide and DHMA 3-sulfate are the major excretion products of MDMA (Figure 1). So far no stereoselective methods for detection and quantification of MDMA and all relevant phase II metabolites in blood plasma are available. Due to their high polarity and therefore low volatility, analysis of phase II metabolites is mainly done by LCMS techniques. For chiral analysis usually chiral HPLC columns are used [40]. However, they are expensive, and their ability to actually separate two enantiomers mainly relies on trial and error. Recent publications managed to separate the stereoisomers of HMMA 4glucuronide using a pirkle brush chiral stationary phase. However MDMA and its other metabolites could not be stereoselectively separated by this method [34]. Other methods separated the enantiomers of MDMA, but no phase II metabolites, on cyclodextrine stationary phases [25]. Chiral derivatization with different chiral derivatization reagents is a commonly used enantioseparation technique for GC-MS analysis. However, in LC-MS analysis derivatization is rather uncommon. Recently, Nakanishi et al described a new LC-MS method after chiral derivatization with $N$-(2,4-dinitrophenyl)-D-leucinamide for stereoselective analysis of MDMA as well as HMMA 4-sulfate and HMMA 4-glucuronide, but not for 
DHMA sulfates in urine [35]. Similar to this method a new method was developed using $N$ (2,4-dinitro-5-fluorophenyl) L-valinamide (DNPV). In this study DNPV was chosen as derivatization reagent as it was commercially available in Europe. The analogue as L- amino acid was prefered over the D-form as it was significantly cheaper. As shown in Figure 2, chemical reaction of DNPV with MDMA and its phase I and II metabolites leads to the formation of diastereomers that can subsequently be separated on standard reversed phase stationary phases.

The analytes included in the method were chosen based on the main metabolites identified in urine [20] and on the measurement of five authentic MDMA blood samples checking for all possible metabolites. In these samples mainly MDMA, HMMA 4-sulfate, HMMA 4glucuronide, and DHMA 3-sulfate could be detected. Minor amounts of MDA, unconjugated HMMA, and DHMA 4-sulfate were present. DHMA and HMA as well as glucuronides of DHMA could not be detected in any of these samples. As DHMA and HMA were commercially available they were nevertheless included into the method.

As the final derivatives are not available as reference material derivatization had to be performed in order to produce these derivatives. Standard MS tuning procedures for MRM transitions did not work from this chemical reaction solvent, probably due to an excess of derivatization reagent still present in the mixture. Therefore determination of MRM transitions for the final method had to be performed manually. For that purpose, methods were written for each analyte targeting the underivatized, onefoldly derivatized and in cases where multiple derivatization was theoretically possible, two- and threefold derivatives. EPI spectra were recorded using a $\mathrm{CE}$ of $10 \mathrm{eV}$ and $40 \mathrm{eV}$, respectively. No differences in the abundances of the fragment ions were observed between the $R$ and $S$ stereoisomers. As exemplarily depicted in Figure 3 for HMMA 4-sulfate-DNPV, from these EPI spectra three fragment ions and a reasonable CE were chosen per analyte for the final MRM method. For all analytes complete derivatization was observed. As derivatized DHMA sulfates were only 
detectable as deconjugated analytes after an in-source fragmentation and loss of the sulfate moiety, the deconjugated analytes were used as precusor ions for the final method. The chosen derivatization procedure lead to the formation of diastereomers that should be separable on a chiral column. Using ammonium acetate (A) and ACN (B) as mobile phase, all analytes were nicely separated into their two stereoisomers, except for DHMA-DNPV. A change in mobile phase composition to ammonium formate and methanol allowed also the separation of DHMA-DNPV isomers, however, resulting in a coelution of MDA-DNPV and DHMA 3-sulfate-DNPV isomers. Also other mobile phase compositions of ammonium formate with $\mathrm{ACN}$, or a mixture of $\mathrm{ACN}$ and methanol did not result in a separation of all analytes. As DHMA was not present in any authentic sample, A and B were finally employed as solvents accepting the coelution of $R$ - and $S$-DHMA-DNPV. The final chromatogram of an extract of an QC MED sample is given in Figure 4. The elution order of the stereoisomers could be determined for MDMA-DNPV and HMMA 4-glucuronide-DNPV using the available $S$-stereoisomer after chiral derivatization. The elution order of all other metabolite diastereomers could be determined by synthesis of the corresponding metabolites by incubation of S-MDMA with HLS9 fractions containing cytochrome P450-, catechol-Omethyltransferase- and sulfotransferase enzymes as principally described in ref. [18]. HMA could not be synthesized from $S$-MDMA in quantitative sufficient amounts. In order to determine its elution order nevertheless, racemic MDA was incubated evaluating the ratio of the two HMA peaks after DNPV derivatization. $S$-MDA was shown to be metabolized preferentially resulting in the larger peaks [41]. With our derivatization procedure, the $R$ stereoisomers of all metabolites were identified to elute before the $S$-stereoisomers, except for MDA-DNPV, HMA-DNPV, and DHMA 4-sulfate-DNPV, where the elution order was reversed. Enantiomer differentiation was essential for determining MDMA's stereoselective pharmacokinetics in blood plasma. 


\section{Method Validation}

The described final procedure was validated according to recommendations on method validation in the context of quality management with forensic-toxicological investigations published by the GTFCh [38] and internationally accepted recommendations [42-46].

\section{Specificity, Selectivity, and Cross Talk}

Blank blood plasma samples from 10 different sources were analyzed for chromatographic interferences. No interfering peaks were detected for any of the analytes in blank samples or after addition of the IS solution.

Furthermore derivatized analytes were analyzed for cross talk and interferences in the MRM transitions of the other analytes. Again, no cross talk for any compound could be detected. This was one of the advantages of using derivatization in LC-MS. Usually, MDMA, MDA, HMMA, and HMA show very similar fragment ion and are prone to cross talk. Through oneand twofold derivatization, fragmentation was altered and resulted in different fragment ions.

\section{Recovery and matrix effects}

RE and ME data are listed in Table 3. All analytes could be extracted with REs over $60 \%$ with acceptable CVs except for the glucuronides. Most probably, the used protein precipitation leads also to co-precipitation of part of the glucuronides. Different extraction procedures such as solid phase extraction or liquid liquid extraction were considered, however due to the large differences in the physico-chemical properties of MDMA and its phase II metabolites, discarded.

No notable MEs were observed for any of the analytes, except for HMMA 4-glucuronideDNPV at QC LOW and for DHMA-DNPV. However, the ME for HMMA 4-glucuronideDNPV was reproducible $(\mathrm{CV}<11 \%)$ and was therefore accepted for pharmacokinetic studies. The sensitivity for DHMA-DNPV was altogether rather low and its detection was only 
possible for QC MED and QC HIGH. Quite a large ME could be detected for DHMA-DNPV in QC HIGH (390\%, CV 60\%) by calculation via the area only. Adjustment via DHBADNPV as IS, which is generally used for quantification, lead to acceptable ME with $91 \%$ $(19 \% \mathrm{CV})$ as given in Table 3.

\section{Calibration model}

Calibration curves using six concentration levels with six replicates each were constructed to evaluate the calibration model. The limits for the calibration curve were assessed based on data published by [47] determined after controlled administration of MDMA. Calibration ranges for all analytes are given in Tables 2 and should allow quantification without further dilution.

A weighted (1/X2) calibration model was used to account for unequal variances (heteroscedasticity) across the calibration range. As there were several possibilities with different ISs and calibration models, the final decision was made after evaluation of the accuracy and precision data. The final calibration model was linear, 1/X2 weighting for all analytes.

\section{Accuracy and precision}

QC samples (LOW, MED, and HIGH) were analyzed in duplicate on each of eight days as was proposed by Peters et al [48]. QC concentrations were determined from daily calibration curves. Calibrator concentrations were within $30 \%$ of target based on the full calibration curve. Accuracy, intra-day and inter-day precision were calculated as described above (Table $3)$.

Respective deuterated analogues were employed for MDA and MDMA. As for all other metabolites no deuterated standards were commercially available, pholedrine was successfully used for HMMA 4-sulfate, HMMA 4-glucuronide, DHMA 3-sulfate, DHMA 4- 
sulfate, HMMA and HMA. DHMA was calculated with DHBA as IS due to its structural similarities.

All analytes fulfilled the validation parameters except for DHMA-DNPV, DHMA 4-sulfateDNPV at QC LOW and HMMA-glucuronide-DNPV at QC LOW. As already mentioned the sensitivity for DHMA-DNPV was rather low, most likely due to the fact that it was threefoldly derivatized. It could be detected from concentrations equal or higher than calibrator 3 and QK MED. Even in QK MED and QK HIGH the inter-day precision was above the acceptance criteria of $+/-15 \%$. However, DHMA was not present in any of the analyzed authentic samples. For DHMA 4-sulfate-DNPV in QC LOW the observed bias was slightly lower than the acceptance criteria and for HMMA 4-glucuronide-DNPV intra- and interday precision was slightly above the acceptance criteria of $+/-20 \%$. No significant differences were observed between $R$ - and $S$-stereoisomer. All together the method was sufficient for pharmacokinetic analysis of authentic samples after a controlled administration of MDMA in blood plasma.

\section{Stability}

No degradation was observed for any derivatized analyte in processed samples stored on the autosampler for $48 \mathrm{~h}$ at ambient temperature. All analytes were stable over three freeze-thaw cycles except for HMA, which was stable over only one cycle. Instability of HMA during freezing and thawing was already described by Kolbrich [49]. Therefore an immediate workup after thawing was recommended [49] which was performed for our samples.

No instability of the phase II analytes after storage at $-20^{\circ} \mathrm{C}$ for 24 months was observed with calculated concentration within $+/-20 \%$ of nominal concentration. Furthermore, no deconjugated analytes could be detected as would be assumed to be formed after degradation of phase II metabolites. Data on MDMA and its phase I metabolites as well as HMMA and 
HMA were previously published and no instability could be observed up to after 6 months $[50]$.

\section{Limit of quantification}

The LOQs of all analytes are listed in Table 2 and were consistent with the lowest calibrator with less than $30 \%$ bias as compared to the target concentration. Those limits were comparable to those published by other authors for MDMA and MDA [23, 49] and were sufficient for the pharmacokinetic analysis in the context of a controlled single MDMA administration study.

\section{Proof of applicability}

The presented method was successfully applied to about 500 samples of a pharmacokinetic study for MDMA following controlled MDMA administration. The data of this study will be presented elsewhere. As shown in Figure 5, stereoselective differences could be seen in blood plasma samples after controlled MDMA administration. 


\section{Conclusion}

A validated LC-MS/MS method for is presented for the simultaneous quantification of MDMA and its relevant phase I and phase II metabolites after chiral derivatization with DNPV in blood plasma. The method fulfilled the required validation criteria for all analytes except for DHMA. The method was successfully applied for the pharmacokinetic study of possible stereoselective phase I and phase II MDMA metabolism after controlled administration of MDMA. 


\section{Acknowledgements}

The authors would like to thank Dr. Markus Baumgartner, Michael Pötzsch and Milena M. Madry for their helpful support and discussion. 


\section{References}

1. J.K. Fallon, A.T. Kicman, J.A. Henry, P.J. Milligan, D.A. Cowan.A.J. Hutt. Stereospecific analysis and enantiomeric disposition of 3, 4methylenedioxymethamphetamine (Ecstasy) in humans. Clin.Chem. 1999, 45, 1058

2. H. Kalant. The pharmacology and toxicology of "ecstasy" (MDMA) and related drugs. CMAJ. 2001, 165, 917

3. S.J. Rietjens, L. Hondebrink, R.H.S. Westerink.J. Meulenbelt. Pharmacokinetics and pharmacodynamics of 3,4-methylenedioxymethamphetamine (MDMA): interindividual differences due to polymorphisms and drug-drug interactions. Critical Reviews in Toxicology. 2012, 42, 854

4. R. de la Torre, M. Farre, P.N. Roset, L.C. Hernandez, M. Mas, J. Ortuno, E. Menoyo, N. Pizarro, J. Segura.J. Cami. Pharmacology of MDMA in humans. Ann.N.Y.Acad.Sci. 2000, 914,225

5. E.M.C.f.D.a.D.A. (EMCDDA). European Drug Report 2013 - Trends and Developments. Lisbon. 2013, 72.

6. U.S.D.o.H.a.H.S.-S.A.a.M.H.S.A.O.o.A. Studies. Results from the 2009, National Survey on Drug Use and Health: Volume I. Summary of National Findings. 2010,

7. T.J. Monks, D.C. Jones, F. Bai.S.S. Lau. The role of metabolism in 3,4-(+)methylenedioxyamphetamine and 3,4-(+)-methylenedioxymethamphetamine (ecstasy) toxicity [review]. Ther.Drug Monit. 2004, 26, 132

8. N. Easton.C.A. Marsden. Ecstasy: are animal data consistent between species and can they translate to humans? [review]. J.Psychopharmacol. 2006, 20, 194

9. U.D. McCann, Z. Szabo, M. Vranesic, M. Palermo, W.B. Mathews, H.T. Ravert, R.F. Dannals.G.A. Ricaurte. Positron emission tomographic studies of brain dopamine and serotonin transporters in abstinent (+/-)3,4-methylenedioxymethamphetamine ("ecstasy") users: relationship to cognitive performance. Psychopharmacology (Berl). 2008, 200, 439

10. F. Bai, S.S. Lau.T.J. Monks. Glutathione and N-acetylcysteine conjugates of alphamethyldopamine produce serotonergic neurotoxicity: possible role in methylenedioxyamphetamine-mediated neurotoxicity. Chem.Res.Toxicol. 1999, 12, 1150 
11. M. Hiramatsu, Y. Kumagai, S.E. Unger.A.K. Cho. Metabolism of methylenedioxymethamphetamine: formation of dihydroxymethamphetamine and a quinone identified as its glutathione adduct. J.Pharmacol.Exp.Ther. 1990, 254, 521

12. R.T. Miller, S.S. Lau.T.J. Monks. 2,5-Bis-(glutathion-S-yl)-alpha-methyldopamine, a putative metabolite of (+/-)-3,4-methylenedioxyamphetamine, decreases brain serotonin concentrations. Eur.J.Pharmacol. 1997, 323, 173

13. M. Mueller, J. Yuan, A. Felim, A. Neudorffer, F.T. Peters, H.H. Maurer, U.D. McCann, M. Largeron.G.A. Ricaurte. Further Studies on the Role of Metabolites in (+/-)-3,4-Methylenedioxymethamphetamine-Induced Serotonergic Neurotoxicity. Drug Metabolism and Disposition. 2009, 37, 2079

14. J.P. Capela, H. Carmo, F. Remiao, M.L. Bastos, A. Meisel.F. Carvalho. Molecular and cellular mechanisms of ecstasy-induced neurotoxicity: an overview. Mol Neurobiol. 2009, 39, 210

15. M. Carvalho, H. Carmo, V.M. Costa, J.P. Capela, H. Pontes, F. Remiao, F. Carvalho.L. Bastos Mde. Toxicity of amphetamines: an update. Arch Toxicol. 2012, 86,1167

16. I. Antolino-Lobo, J. Meulenbelt, S.M. Nijmeijer, P. Scherpenisse, M. van den Berg.M.B.M. van Duursen. Differential Roles of Phase I and Phase II Enzymes in 3,4Methylendioxymethamphetamine-Induced Cytotoxicity. Drug Metabolism and Disposition. 2010, 38, 1105

17. H.H. Maurer, J. Bickeboeller-Friedrich, T. Kraemer.F.T. Peters. Toxicokinetics and analytical toxicology of amphetamine-derived designer drugs ("Ecstasy").

Toxicol.Lett. 2000, 112, 133

18. H.H. Maurer. On the metabolism and the toxicological analysis of methylenedioxyphenylalkylamine designer drugs by gas chromatography-mass spectrometry. Ther.Drug Monit. 1996, 18, 465

19. R. de la Torre, M. Farre, P.N. Roset, N. Pizarro, S. Abanades, M. Segura, J. Segura.J. Cami. Human pharmacology of MDMA: pharmacokinetics, metabolism, and disposition [review]. Ther.Drug Monit. 2004, 26, 137

20. A.E. Schwaninger, M.R. Meyer, A.J. Barnes, E.A. Kolbrich-Spargo, D.A. Gorelick, R.S. Goodwin, M.A. Huestis.H.H. Maurer. Urinary excretion kinetics of 3,4methylenedioxymethamphetamine (MDMA, ecstasy) and its phase I and phase II 
metabolites in humans following controlled MDMA administration. Clin Chem. 2011, 57,1748

21. T. Kraemer.H.H. Maurer. Toxicokinetics of amphetamines: Metabolism and toxicokinetic data of designer drugs, of amphetamine, methamphetamine and their $\mathrm{N}$ alkyl derivatives [review]. Ther.Drug Monit. 2002, 24, 277

22. F.T. Peters, N. Samyn, M. Wahl, T. Kraemer, G. de Boeck.H.H. Maurer. Concentrations and Ratios of Amphetamine, Methamphetamine, MDA, MDMA, and MDEA Enantiomers determined in Plasma Samples From Clinical Toxicology and Driving Under the Influence of Drugs Cases by GC-NICI-MS. J.Anal.Toxicol. 2003, 27,552

23. F.T. Peters, N. Samyn, C. Lamers, W. Riedel, T. Kraemer, G. de Boeck.H.H. Maurer. Drug Testing in Blood: Validated Negative-Ion Chemical Ionization Gas Chromatographic-Mass Spectrometric Assay for Enantioselective Determination of the Designer Drugs MDA, MDMA (Ecstasy) and MDEA and Its Application to Samples from a Controlled Study with MDMA. Clin.Chem. 2005, 51, 1811

24. N. Pizarro, M. Farre, M. Pujadas, A.M. Peiro, P.N. Roset, J. Joglar.R. de la Torre. Stereochemical analysis of 3,4-methylenedioxymethamphetamine and its main metabolites in human samples including the catechol-type metabolite $(3,4-$ dihydroxymethamphetamine). Drug Metab.Dispos. 2004, 32, 1001

25. M.R. Meyer, F.T. Peters.H.H. Maurer. The Role of Human Hepatic Cytochrome P450 Isozymes in the Metabolism of Racemic MDMA and its Enantiomers. Drug Metab.Dispos. 2008, 36, 2345

26. M.R. Meyer.H.H. Maurer. Enantioselectivity in the methylation of the catecholic phase I metabolites of methylenedioxy designer drugs and their capability to inhibit catechol-O-methyltransferase-catalyzed dopamine 3-methylation. Chem Res Toxicol. 2009, 22, 1205

27. A.E. Schwaninger, M.R. Meyer.H.H. Maurer. Investigation on the enantioselectivity of the sulfation of the methylenedioxymethamphetamine metabolites 3,4dihydroxymethamphetamine and 4-hydroxy-3-methoxymethamphetamine using the substrate-depletion approach. Drug Metab Dispos. 2011, 39, 1998

28. A.E. Schwaninger, M.R. Meyer, J. Zapp.H.H. Maurer. The role of human UDPglucuronyltransferases on the formation of the methylenedioxymethamphetamine (ecstasy) phase II metabolites R- and S-3-methoxymethamphetamine 4-Oglucuronides. Drug Metab Dispos. 2009, 37, 2212 
29. F.T. Peters, S. Schaefer, R.F. Staack, T. Kraemer.H.H. Maurer. Screening for and validated quantification of amphetamines and of amphetamine- and piperazine-derived designer drugs in human blood plasma by gas chromatography/mass spectrometry. J.Mass Spectrom. 2003, 38, 659

30. N. Pizarro, A. Llebaria, S. Cano, J. Joglar, M. Farre, J. Segura.R. de la Torre. Stereochemical analysis of 3,4-methylenedioxymethamphetamine and its main metabolites by gas chromatography/mass spectrometry. Rapid Commun.Mass Spectrom. 2003, 17, 330

31. W.A.W. Raihana, S.H. Gan.S.C. Tan. Stereoselective method development and validation for determination of concentrations of amphetamine-type stimulants and metabolites in human urine using a simultaneous extraction-chiral derivatization approach. Journal of Chromatography B-Analytical Technologies in the Biomedical and Life Sciences. 2011, 879, 8

32. D. Hensley.J.T. Cody. Simultaneous determination of amphetamine, methamphetamine, methylenedioxyamphetamine (MDA), methylenedioxymethamphetamine (MDMA), and methylenedioxyethylamphetamine (MDEA) enantiomers by GC-MS. J.Anal.Toxicol. 1999, 23, 518

33. N. Shima, M. Katagi, H. Kamata, K. Zaitsu, T. Kamata, M. Nishikawa, A. Miki, H. Tsuchihashi, T. Sakuma.N. Nemoto. Urinary excretion of the main metabolites of 3,4methylenedioxymethamphetamine (MDMA), including the sulfate and glucuronide of 4-hydroxy-3-methoxymethamphetamine (HMMA), in humans and rats. Xenobiotica. 2008, 38,314

34. A.E. Schwaninger, M.R. Meyer, M.A. Huestis.H.H. Maurer. Development and validation of LC-HRMS and GC-NICI-MS methods for stereoselective determination of MDMA and its phase I and II metabolites in human urine. J Mass Spectrom. 2011, 46,603

35. K. Nakanishi, M. Katagi, K. Zaitsu, N. Shima, H. Kamata, A. Miki, H. Kato, K.I. Harada, H. Tsuchihashi.K. Suzuki. Simultaneous enantiomeric determination of MDMA and its phase I and phase II metabolites in urine by liquid chromatographytandem mass spectrometry with chiral derivatization. Anal Bioanal Chem. 2012, 404, 2427

36. A.E. Schwaninger, M.R. Meyer, J. Zapp.H.H. Maurer. Sulfation of the 3,4methylenedioxymethamphetamine (MDMA) metabolites 3,4dihydroxymethamphetamine (DHMA) and 4-hydroxy-3-methoxymethamphetamine (HMMA) and their capability to inhibit human sulfotransferases. Toxicol Lett. 2011, 202,120 
37. B.K. Matuszewski, M.L. Constanzer.C.M. Chavez-Eng. Strategies for the assessment of matrix effect in quantitative bioanalytical methods based on HPLC-MS/MS. Anal. Chem. 2003, 75, 3019

38. F.T. Peters, M. Hartung, M. Herbold, G. Schmitt, T. Daldrup.F. Musshoff. Anhang B zur Richtlinie der GTFCh zur Qualitätssicherung bei forensisch-toxikologischen Untersuchungen. Anforderungen an die Validierung von Analysenmethoden. Toxichem. Krimtech. 2009, 76, 185

39. F.T. Peters, N. Samyn, T. Kraemer, G. de Boeck.H.H. Maurer. Enantioselective determination of amphetamine, methamphetamine, MDA and MDMA in plasma samples from clinical toxicology and driving under the influence of drugs cases using GC-NICI-MS [abstract]. Ther.Drug Monit. 2003, 25, 539

40. A.E. Schwaninger, M.R. Meyer.H.H. Maurer. Chiral drug analysis using mass spectrometric detection relevant to research and practice in clinical and forensic toxicology. Journal of Chromatography A. 2012, 1269, 122

41. M.R. Meyer, F.T. Peters.H.H. Maurer. Investigations on the human hepatic cytochrome P450 isozymes involved in the metabolism of 3,4-methylenedioxyamphetamine (MDA) and benzodioxolyl-butanamine (BDB) enantiomers. Toxicology Letters. 2009, 190, 54

42. V.P. Shah, K.K. Midha, S. Dighe, I.J. McGilveray, J.P. Skelly, A. Yacobi, T. Layloff, C.T. Viswanathan, C.E. Cook, R.D. McDowall, K.A. Pittman.S. Spector. Analytical methods validation: bioavailability, bioequivalence and pharmacokinetic studies. Conference report. Pharm.Res. 1992, 9, 588

43. V.P. Shah, K.K. Midha, J.W. Findlay, H.M. Hill, J.D. Hulse, I.J. McGilveray, G. McKay, K.J. Miller, R.N. Patnaik, M.L. Powell, A. Tonelli, C.T. Viswanathan.A. Yacobi. Bioanalytical method validation - a revisit with a decade of progress. Pharm.Res. 2000, 17, 1551

44. W. Lindner.I.W. Wainer. Requirements for initial assay validation and publication in J. Chromatography B [editorial]. J.Chromatogr.B. 1998, 707, 1

45. Evaluation of Precision Performance of Clinical Chemistry Devices; Approved Guideline. 1999

46. F.T. Peters.H.H. Maurer. Bioanalytical method validation and its implications for forensic and clinical toxicology - A review [review]. Accred.Qual.Assur. 2002, 7, 441 
47. E.A. Kolbrich, R.S. Goodwin, D.A. Gorelick, R.J. Hayes, E.A. Stein.M.A. Huestis. Plasma pharmacokinetics of 3,4-methylenedioxymethamphetamine after controlled oral administration to young adults. Ther.Drug Monit. 2008, 30, 320

48. F.T. Peters. Method Validation using LC-MS. London. 2006, 71

49. E.A. Kolbrich, R.H. Lowe.M.A. Huestis. Two-Dimensional Gas Chromatography/Electron-Impact Mass Spectrometry with Cryofocusing for Simultaneous Quantification of MDMA, MDA, HMMA, HMA, and MDEA in Human Plasma. Clin.Chem. 2008, 54, 379

50. K.M. Clauwaert, J.F. Van Bocxlaer.A.P. De Leenheer. Stability study of the designer drugs "MDA, MDMA and MDEA" in water, serum, whole blood, and urine under various storage temperatures. Forensic Sci.Int. 2001, 124, 36 


\section{Legend to Figures}

Figure 1: Main metabolic pathways of MDMA

Figure 2: Chiral derivatization with $N$-(2,4-dinitro-5-fluorophenyl) L-valinamide (DNPV) exemplified for HMMA 4-sulfate for onefold derivatization (A) and for HMMA for twofold derivatization $(\mathrm{B})$

Figure 3: Evaluation and optimization of MS parameters for MRM quantification. A: EPI spectrum of HMMA 4-sulfate-DNPV at $10 \mathrm{eV}$ collision energy (CE), B: EPI spectrum of HMMA 4-sulfate-DNPV at $40 \mathrm{eV} \mathrm{CE}$. The dotted lines in the molecular formula represent the fragmentation pattern of the fragments depicted in the EPI spectra at 10 (A) and $40 \mathrm{eV}(\mathrm{B})$. From the EPI spectra MRM transitions were optimized for quantification of HMMA 4sulfate-DNPV(C)

Figure 4: Typical MRM chromatograms and elution order of derivatized $R$ and $S$ stereoisomers of MDMA and all monitored phase I and phase II metabolites at QC med concentration.

Figure 5: MRM chromatogram of an authentic derivatized extract of a human blood plasma sample at $t_{\max }$ after ingestion of MDMA 
Tables

Table 1: MS settings and retention times of the measured analytes sorted by elution order. Quantifiers are given in bold print.

\begin{tabular}{|c|c|c|c|c|c|c|c|}
\hline 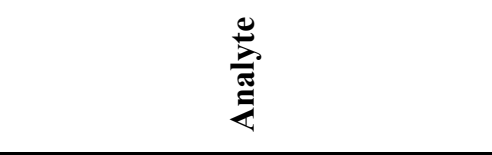 & $\begin{array}{l}\tilde{E} \\
\tilde{\Xi} \\
\bar{\sigma}\end{array}$ & 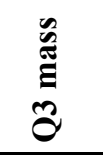 & $\underline{\underline{\simeq}}$ & $\overrightarrow{\hat{a}}$ & $\vec{a}$ & $\begin{array}{l}\text { zo } \\
\text { si }\end{array}$ & $\overrightarrow{\hat{x}}$ \\
\hline \multirow{3}{*}{$R$-HMMA 4-glucuronide DNPV } & 652.0 & 165.0 & 13.7 & 100 & 10 & 30 & 12 \\
\hline & 652.0 & 476.0 & 13.7 & 100 & 10 & 15 & 12 \\
\hline & 652.0 & 137.0 & 13.7 & 100 & 10 & 30 & 12 \\
\hline \multirow{3}{*}{$S$-HMMA 4-glucuronide DNPV } & 652.1 & 165.0 & 14.1 & 100 & 10 & 30 & 12 \\
\hline & 652.1 & 476.0 & 14.1 & 100 & 10 & 15 & 12 \\
\hline & 652.1 & 137.0 & 14.1 & 100 & 10 & 30 & 12 \\
\hline \multirow{3}{*}{$R$-HMMA 4-sulfate DNPV } & 556.0 & 165.0 & 14.9 & 100 & 10 & 30 & 12 \\
\hline & 556.0 & 137.0 & 14.9 & 100 & 10 & 30 & 12 \\
\hline & 556.0 & 476.0 & 14.9 & 100 & 10 & 15 & 12 \\
\hline \multirow{3}{*}{$S$-HMMA 4-sulfate DNPV } & 556.1 & 165.0 & 15.8 & 100 & 10 & 30 & 12 \\
\hline & 556.1 & 137.0 & 15.8 & 100 & 10 & 30 & 12 \\
\hline & 556.1 & 476.0 & 15.8 & 100 & 10 & 15 & 12 \\
\hline \multirow{3}{*}{$S$-DHMA 4-sulfate 2 DNPV } & 742.0 & 386.0 & 19.2 & 100 & 10 & 30 & 12 \\
\hline & 742.0 & 293.0 & 19.2 & 100 & 10 & 30 & 12 \\
\hline & 742.0 & 328.0 & 19.2 & 100 & 10 & 30 & 12 \\
\hline \multirow{3}{*}{$R$-DHMA 4-sulfate 2 DNPV } & 742.1 & 386.0 & 19.6 & 100 & 10 & 30 & 12 \\
\hline & 742.1 & 293.0 & 19.6 & 100 & 10 & 30 & 12 \\
\hline & 742.1 & 328.0 & 19.6 & 100 & 10 & 30 & 12 \\
\hline \multirow{3}{*}{$R$-DHMA 3-sulfate 2 DNPV } & 742.0 & 386.1 & 19.8 & 100 & 10 & 30 & 12 \\
\hline & 742.0 & 293.1 & 19.8 & 100 & 10 & 30 & 12 \\
\hline & 742.0 & 328.1 & 19.8 & 100 & 10 & 30 & 12 \\
\hline \multirow{3}{*}{$S$-DHMA 3-sulfate 2 DNPV } & 742.1 & 386.1 & 20.0 & 100 & 10 & 30 & 12 \\
\hline & 742.1 & 293.1 & 20.0 & 100 & 10 & 30 & 12 \\
\hline & 742.1 & 328.1 & 20.0 & 100 & 10 & 30 & 12 \\
\hline$R$-MDMA d5 DNPV & 479.0 & 165.0 & 20.4 & 100 & 10 & 19 & 12 \\
\hline \multirow{3}{*}{$R$-MDMA DNPV } & 474.0 & 163.2 & 20.4 & 100 & 10 & 19 & 12 \\
\hline & 474.0 & 104.9 & 20.4 & 100 & 10 & 67 & 14 \\
\hline & 474.0 & 135.0 & 20.4 & 100 & 10 & 55 & 8 \\
\hline$S$-MDMA d5 DNPV & 479.1 & 165.0 & 20.9 & 100 & 10 & 19 & 12 \\
\hline \multirow{3}{*}{$S$-MDMA DNPV } & 474.1 & 163.2 & 20.9 & 100 & 10 & 19 & 12 \\
\hline & 474.1 & 104.9 & 20.9 & 100 & 10 & 67 & 14 \\
\hline & 474.1 & 135.0 & 20.9 & 100 & 10 & 55 & 8 \\
\hline$S$-MDA d5 DNPV & 465.0 & 110.0 & 21.5 & 100 & 10 & 67 & 14 \\
\hline \multirow{3}{*}{$S$-MDA DNPV } & 460.0 & 163.0 & 21.5 & 100 & 10 & 19 & 12 \\
\hline & 460.0 & 105.0 & 21.5 & 100 & 10 & 67 & 14 \\
\hline & 460.0 & 135.0 & 21.5 & 100 & 10 & 55 & 8 \\
\hline$R$-MDA d5 DNPV & 465.1 & 110.0 & 22.0 & 100 & 10 & 67 & 14 \\
\hline \multirow{3}{*}{$R$-MDA DNPV } & 460.1 & 163.0 & 22.0 & 100 & 10 & 19 & 12 \\
\hline & 460.1 & 105.0 & 22.0 & 100 & 10 & 67 & 14 \\
\hline & 460.1 & 135.0 & 22.0 & 100 & 10 & 55 & 8 \\
\hline \multirow{3}{*}{$R$-HMMA 2 DNPV } & 756.0 & 400.0 & 23.2 & 100 & 10 & 30 & 12 \\
\hline & 756.0 & 293.0 & 23.2 & 100 & 10 & 30 & 12 \\
\hline & 756.0 & 342.0 & 23.2 & 100 & 10 & 30 & 12 \\
\hline
\end{tabular}




\begin{tabular}{|c|c|c|c|c|c|c|c|}
\hline 1-Pholedrine 2 DNPV & 726.0 & 370.0 & 23.5 & 100 & 10 & 30 & 12 \\
\hline \multirow{3}{*}{$S$-HMMA 2 DNPV } & 756.1 & 400.0 & 23.7 & 100 & 10 & 30 & 12 \\
\hline & 756.1 & 293.0 & 23.7 & 100 & 10 & 30 & 12 \\
\hline & 756.1 & 342.0 & 23.7 & 100 & 10 & 30 & 12 \\
\hline \multirow[t]{2}{*}{2 Pholedrine DNPV } & 726.1 & 370.0 & 23.9 & 100 & 10 & 30 & 12 \\
\hline & 742.0 & 279.0 & 24.1 & 100 & 10 & 30 & 12 \\
\hline \multirow[t]{2}{*}{1 HMA 2 DNPV } & 742.0 & 234.0 & 24.1 & 100 & 10 & 30 & 12 \\
\hline & 742.0 & 400.0 & 24.1 & 100 & 10 & 30 & 12 \\
\hline \multirow[t]{2}{*}{ DHBA 3 DNPV } & 980.0 & 655.0 & 24.4 & 100 & 10 & 30 & 12 \\
\hline & 1022.0 & 293.0 & 24.8 & 100 & 10 & 30 & 12 \\
\hline \multirow[t]{3}{*}{ DHMA 3 DNPV } & 1022.0 & 397.0 & 24.8 & 100 & 10 & 30 & 12 \\
\hline & 1022.0 & 621.0 & 24.8 & 100 & 10 & 30 & 12 \\
\hline & 742.1 & 279.0 & 25.0 & 100 & 10 & 30 & 12 \\
\hline \multirow[t]{2}{*}{2 HMA 2 DNPV } & 742.1 & 234.0 & 25.0 & 100 & 10 & 30 & 12 \\
\hline & 742.1 & 400.0 & 25.0 & 100 & 10 & 30 & 12 \\
\hline
\end{tabular}


Table 2: Blood plasma concentrations for calibrator (cal) and quality control (QC) samples used for method validation

\begin{tabular}{|c|c|c|c|c|c|c|c|c|c|}
\hline \multirow[b]{2}{*}{ Analyte } & \multicolumn{9}{|c|}{ Blood plasma concentration $\mu \mathrm{M}(\mu \mathrm{g} / \mathrm{L})$} \\
\hline & Cal. 1 & Cal. 2 & Cal. 3 & Cal. 4 & Cal. 5 & Cal. 6 & QC low & QC med & QC high \\
\hline$R$-HMMA 4-glucuronide DNPV & $\mathbf{0 . 0 0 2 5}(0.93)$ & $\mathbf{0 . 0 5}(18.6)$ & $\mathbf{0 . 2 5}(92.8)$ & $0.5(185)$ & $0.75(278)$ & $1.25(464)$ & $\mathbf{0 . 0 0 3 7 5}(1.4)$ & $\mathbf{0 . 3 7 5}(139)$ & $1.0(371)$ \\
\hline$S$-HMMA 4-glucuronide DNPV & $\mathbf{0 . 0 0 2 5}(0.93)$ & $\mathbf{0 . 0 5}(18.6)$ & $\mathbf{0 . 2 5}(92.8)$ & $0.5(185)$ & $\mathbf{0 . 7 5}(278)$ & $1.25(464)$ & $0.00375(1.4)$ & 0.375 (139) & $\mathbf{1 . 0}(371)$ \\
\hline$R$-HMMA 4-sulfate DNPV & $\mathbf{0 . 0 0 2 5}(0.69)$ & $\mathbf{0 . 0 5}(13.8)$ & $\mathbf{0 . 2 5}(68.8)$ & $0.5(138)$ & $\mathbf{0 . 7 5}(206)$ & $1.0(275)$ & $0.00375(1.0)$ & $\mathbf{0 . 3 7 5}(103)$ & $\mathbf{0 . 7}(193)$ \\
\hline$S$-HMMA 4-sulfate DNPV & $\mathbf{0 . 0 0 2 5}(0.69)$ & $\mathbf{0 . 0 5}(13.8)$ & $\mathbf{0 . 2 5}(68.8)$ & $0.5(138)$ & $\mathbf{0 . 7 5}(206)$ & $\mathbf{1 . 0}(275)$ & $\mathbf{0 . 0 0 3 7 5}(1.0)$ & $\mathbf{0 . 3 7 5}(103)$ & $0.7(193)$ \\
\hline$S$-DHMA 4-sulfate 2 DNPV & $0.0025(0.65)$ & $\mathbf{0 . 0 5}(13.1)$ & $0.25(65.3)$ & $0.5(131)$ & $0.75(196)$ & & $\mathbf{0 . 0 0 3 7 5}(0.98)$ & $0.375(98.8)$ & $0.6(157)$ \\
\hline$R$-DHMA 4-sulfate 2 DNPV & $\mathbf{0 . 0 0 2 5}(0.65)$ & $\mathbf{0 . 0 5}(13.1)$ & $0.25(65.3)$ & $0.5(131)$ & $\mathbf{0 . 7 5}(196)$ & & $\mathbf{0 . 0 0 3 7 5}(0.98)$ & $\mathbf{0 . 3 7 5}(98.8)$ & $0.6(157)$ \\
\hline$R$-DHMA 3-sulfate 2 DNPV & $\mathbf{0 . 0 0 2 5}(0.65)$ & $\mathbf{0 . 0 5}(13.1)$ & $0.25(65.3)$ & $0.5(131)$ & $0.75(196)$ & $\mathbf{1 . 0}(261)$ & $\mathbf{0 . 0 0 3 7 5}(0.98)$ & $\mathbf{0 . 3 7 5}(98.8)$ & $0.7(183)$ \\
\hline$S$-DHMA 3-sulfate 2 DNPV & $\mathbf{0 . 0 0 2 5}(0.65)$ & $\mathbf{0 . 0 5}(13.1)$ & $0.25(65.3)$ & $0.5(131)$ & $\mathbf{0 . 7 5}(196)$ & $\mathbf{1 . 0}(261)$ & $\mathbf{0 . 0 0 3 7 5}(0.98)$ & $\mathbf{0 . 3 7 5}(98.8)$ & $0.7(183)$ \\
\hline$R$-MDMA DNPV & $\mathbf{0 . 0 0 2 5}(0.48)$ & $\mathbf{0 . 0 5}(9.6)$ & $0.25(48.3)$ & $\mathbf{0 . 5}(96.5)$ & $0.75(145)$ & $1.25(241)$ & $\mathbf{0 . 0 0 3 7 5}(0.72)$ & $0.375(72.4)$ & $1.0(193)$ \\
\hline$S$-MDMA DNPV & $\mathbf{0 . 0 0 2 5}(0.48)$ & $\mathbf{0 . 0 5}(9.6)$ & $0.25(48.3)$ & $\mathbf{0 . 5}(96.5)$ & $0.75(145)$ & $1.25(241)$ & $\mathbf{0 . 0 0 3 7 5}(0.72)$ & $0.375(72.4)$ & $1.0(193)$ \\
\hline$S$-MDA DNPV & $\mathbf{0 . 0 0 2 5}(0.45)$ & $\mathbf{0 . 0 0 5}(0.90)$ & $0.025(4.5)$ & $\mathbf{0 . 0 5}(9.0)$ & $\mathbf{0 . 1 2 5}(22.4)$ & $0.25(44.8)$ & $\mathbf{0 . 0 0 3 7 5}(0.66)$ & $\mathbf{0 . 0 3 7 5}(6.7)$ & $0.2(35.8)$ \\
\hline$R$-MDA DNPV & $\mathbf{0 . 0 0 2 5}(0.45)$ & $\mathbf{0 . 0 0 5}(0.90)$ & $0.025(4.5)$ & $\mathbf{0 . 0 5}(9.0)$ & $0.125(22.4)$ & $0.25(44.8)$ & $\mathbf{0 . 0 0 3 7 5}(0.66)$ & $\mathbf{0 . 0 3 7 5}(6.7)$ & $0.2(35.8)$ \\
\hline$R$-HMMA 2 DNPV & $\mathbf{0 . 0 0 2 5}(0.49)$ & $\mathbf{0 . 0 0 5}(0.98)$ & $\mathbf{0 . 0 2 5}(4.9)$ & $\mathbf{0 . 0 5}(9.8)$ & $0.125(24.4)$ & $0.25(48.8)$ & $\mathbf{0 . 0 0 3 7 5}(0.73)$ & $\mathbf{0 . 0 3 7 5}(7.3)$ & $0.2(39.0)$ \\
\hline$S$-HMMA 2 DNPV & $\mathbf{0 . 0 0 2 5}(0.49)$ & $\mathbf{0 . 0 0 5}(0.98)$ & $\mathbf{0 . 0 2 5}(4.9)$ & $\mathbf{0 . 0 5}(9.8)$ & $0.125(24.4)$ & $0.25(48.8)$ & $\mathbf{0 . 0 0 3 7 5}(0.73)$ & $\mathbf{0 . 0 3 7 5}(7.3)$ & $0.2(39.0)$ \\
\hline 1 HMA 2 DNPV & $\mathbf{0 . 0 0 2 5}(0.45)$ & $\mathbf{0 . 0 0 5}(0.91)$ & $\mathbf{0 . 0 2 5}(4.5)$ & $\mathbf{0 . 0 5}(9.1)$ & $0.125(22.6)$ & $0.25(45.3)$ & $\mathbf{0 . 0 0 3 7 5}(0.68)$ & $\mathbf{0 . 0 3 7 5}(6.8)$ & $0.2(36.0)$ \\
\hline 2 HMA 2 DNPV & $\mathbf{0 . 0 0 2 5}(0.45)$ & $\mathbf{0 . 0 0 5}(0.91)$ & $\mathbf{0 . 0 2 5}(4.5)$ & $\mathbf{0 . 0 5}(9.1)$ & $0.125(22.6)$ & $0.25(45.3)$ & $\mathbf{0 . 0 0 3 7 5}(0.68)$ & $\mathbf{0 . 0 3 7 5}(6.8)$ & $0.2(36.0)$ \\
\hline DHMA 3 DNPV & $\mathbf{0 . 0 0 5}(0.91)$ & $\mathbf{0 . 0 1}(1.8)$ & $\mathbf{0 . 0 5}(9.1)$ & $\mathbf{0 . 1}(18.1)$ & $0.25(45.3)$ & $0.5(90.5)$ & $0.0075(1.4)$ & $\mathbf{0 . 0 7 5}(13.6)$ & $0.4(72.4)$ \\
\hline
\end{tabular}


Table 3: Method validation data: bias, intra-day precision $\left(\mathrm{RSD}_{\mathrm{R}}\right)$, interday-precision $\left(\mathrm{RSD}_{\mathrm{T}}\right)$, recovery $(\mathrm{RE})$, and matrix effects $(\mathrm{ME})$

\begin{tabular}{|c|c|c|c|c|c|c|c|c|c|c|c|c|c|}
\hline \multirow[b]{2}{*}{ 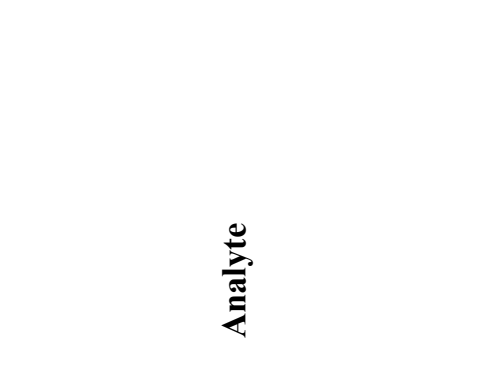 } & \multicolumn{5}{|c|}{ QC low } & \multicolumn{3}{|c|}{ QC med } & \multicolumn{5}{|c|}{ QC high } \\
\hline & $\begin{array}{l}\partial^{0} \\
\dot{\tilde{n}} \\
\dot{\omega}\end{array}$ & $\begin{array}{l}\partial^{\circ} \\
\tilde{\hat{n}} \\
\tilde{\approx}\end{array}$ & $\begin{array}{l}\partial^{\circ} \\
\hat{0} \\
\stackrel{0}{\simeq}\end{array}$ & 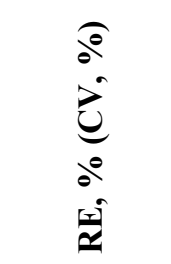 & 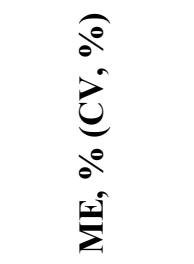 & 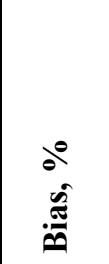 & $\begin{array}{l}0^{0} \\
\hat{n}^{2}\end{array}$ & $\begin{array}{l}\hat{0}^{0} \\
\hat{0} \\
\tilde{0}\end{array}$ & 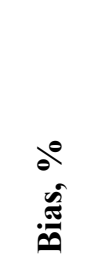 & $\begin{array}{l}\dot{0}^{\circ} \\
\stackrel{\tilde{n}}{\simeq}\end{array}$ & $\begin{array}{l}\partial^{0} \\
\hat{\sigma} \\
\hat{\theta}\end{array}$ & 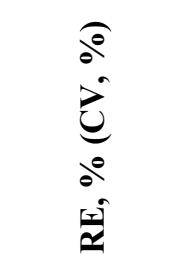 & 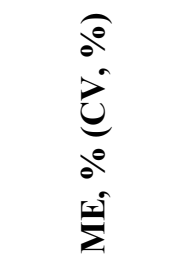 \\
\hline$R$-HMMA 4-glucuronide DNPV & -0.9 & 24.6 & 24.6 & $43.6(20.3)$ & $129.5(11.0)$ & 4.8 & 7.4 & 14.2 & -2.3 & 11.3 & 12.6 & $42.9(15.1)$ & $99.9(4.4)$ \\
\hline$S$-HMMA 4-glucuronide DNPV & 9.5 & 29.9 & 27.7 & $48.2(20.5)$ & $126.3(8.6)$ & -1.7 & 9.7 & 13.2 & 2.9 & 11.5 & 12.3 & $42.7(18.3)$ & $101.0(3.8)$ \\
\hline$R$-HMMA 4-sulfate DNPV & -9.0 & 10.5 & 15.0 & $84.7(9.9)$ & $96.8(4.3)$ & -9.8 & 6.0 & 8.7 & 0.9 & 10.9 & 11.1 & $79.3(4.5)$ & $94.5(3.4)$ \\
\hline$S$-HMMA 4-sulfate DNPV & -9.6 & 10.7 & 14.6 & $81.9(6.7)$ & $97.8(2.4)$ & -9.5 & 7.3 & 9.1 & -0.4 & 9.6 & 9.8 & $78.9(4.8)$ & $91.5(5.5)$ \\
\hline$S$-DHMA 4-sulfate 2 DNPV & -30.0 & 1.1 & 3.9 & $78.9(13.3)$ & $94.6(11.8)$ & -0.6 & 6.9 & 7.3 & -6.0 & 10.9 & 10.8 & $79.3(8.8)$ & $84.8(7.7)$ \\
\hline$R$-DHMA 4-sulfate 2 DNPV & -31.2 & 1.1 & 2.8 & $63.9(9.5)$ & $101.9(10.5)$ & 0.0 & 7.0 & 7.4 & -5.0 & 10.2 & 10.5 & $79.8(8.5)$ & $85.0(5.6)$ \\
\hline$R$-DHMA 3-sulfate 2 DNPV & $\begin{array}{ll}-19.8 \\
\end{array}$ & 13.1 & 13.2 & $79.4(15.5)$ & $95.7(5.7)$ & -6.2 & 7.9 & 7.5 & -1.6 & 10.4 & 10.2 & $83.3(9.1)$ & $80.7(6.4)$ \\
\hline$S$-DHMA 3-sulfate 2 DNPV & -16.8 & 7.3 & 13.5 & $77.8(15.9)$ & $93.6(5.5)$ & -4.5 & 8.0 & 7.6 & -0.9 & 12.8 & 12.4 & $80.7(8.5)$ & $84.7(6.2)$ \\
\hline$R$-MDMA DNPV & -12.5 & 5.9 & 10.9 & $86.1(6.9)$ & $96.8(7.9)$ & -11.1 & 4.7 & 5.7 & -9.1 & 7.0 & 7.3 & $84.2(12.2)$ & $100.9(4.0)$ \\
\hline$S$-MDMA DNPV & -9.4 & 10.9 & 13.5 & $88.8(10.8)$ & $95.8(7.7)$ & -11.6 & 5.7 & 6.4 & -8.1 & 6.0 & 7.1 & $85.8(12.0)$ & $101.9(3.6)$ \\
\hline$S$-MDA DNPV & 10.8 & 7.4 & 12.2 & $83.9(10.6)$ & $92.2(6.5)$ & -5.3 & 6.5 & 7.8 & $\begin{array}{ll}-1.8 \\
\end{array}$ & 7.2 & 8.4 & $85.5(12.3)$ & $93.0(8.0)$ \\
\hline$R$-MDA DNPV & 8.0 & 8.9 & 13.5 & $86.9(9.4)$ & $92.9(6.7)$ & -4.8 & 5.8 & 7.8 & -0.9 & 8.6 & 9.2 & $83.4(10.7)$ & $95.4(5.3)$ \\
\hline$R$-HMMA 2 DNPV & -4.7 & 5.5 & 5.9 & $88.7(9.5)$ & $112.0(5.2)$ & -9.7 & 4.2 & 5.5 & -10.4 & 5.8 & 7.1 & $83.9(8.0)$ & $108.3(5.2)$ \\
\hline$S$-HMMA 2 DNPV & -9.6 & 7.6 & 8.3 & $90.4(12.4)$ & $125.1(4.8)$ & -7.5 & 4.2 & 5.8 & -7.8 & 7.3 & 7.8 & $85.0(7.5)$ & $112.8(3.4)$ \\
\hline 1 HMA 2 DNPV & -17.2 & 4.9 & 7.7 & $81.1(8.2)$ & $118.1(6.6)$ & -14.7 & 4.0 & 4.8 & -9.5 & 6.8 & 8.4 & $80.6(5.6)$ & $98.0(9.5)$ \\
\hline 2 HMA 2 DNPV & -15.0 & 4.7 & 7.9 & $58.7(15.2)$ & $99.8(20.8)$ & -15.5 & 5.1 & 5.3 & -10.6 & 7.2 & 8.0 & $79.8(7.5)$ & $95.1(5.9)$ \\
\hline DHMA 3 DNPV & - & - & - & - & - & 13.4 & 6.0 & 26.1 & -7.8 & 15.2 & 22.9 & $79.9(14.7)$ & $90.6(18.8)^{*}$ \\
\hline
\end{tabular}

* calculated using the ratio analyte/IS 